Research Article

\title{
The Constants in A Posteriori Error Indicator for State-Constrained Optimal Control Problems with Spectral Methods
}

\author{
Jianwei Zhou \\ Department of Mathematics, Linyi University, Shandong, China \\ Correspondence should be addressed to Jianwei Zhou; jwzhou@yahoo.com
}

Received 15 January 2014; Revised 7 April 2014; Accepted 26 April 2014; Published 19 May 2014

Academic Editor: Elena Berdysheva

Copyright (c) 2014 Jianwei Zhou. This is an open access article distributed under the Creative Commons Attribution License, which permits unrestricted use, distribution, and reproduction in any medium, provided the original work is properly cited.

We employ Legendre-Galerkin spectral methods to solve state-constrained optimal control problems. The constraint on the state variable is an integration form. We choose one-dimensional case to illustrate the techniques. Meanwhile, we investigate the explicit formulae of constants within a posteriori error indicator.

\section{Introduction}

Spectral methods provide higher accurate approximations with a relatively small number of unknowns and play increasingly important roles in design optimization, engineering design, and other scientific and engineering computations. Gottlieb and Orszag [1] summarized the theories and applications of spectral methods. There have been extensive researches on finite element methods for optimal control problems, most of which focus on control-constrained problems; see [2-5]. The authors studied the optimal control problems with the control constraint with spectral methods in [6]. In applications of engineering, one cares more about how to control the average value or $L^{2}$-norms of the state variable. The authors [7] discussed state-constrained optimal control problems with finite element methods. However, there are few work on the state-constrained optimal control problems with spectral methods.

In order to get a numerical solution with acceptable accuracy, spectral methods only increase the degree of basis when the error indicator is larger than the a posteriori error indicator, while the finite element methods refine meshes (see $[8,9])$. There have been lots of papers on the a posteriori error estimates for h-version finite element methods but not for spectral methods. Guo [10] got a reliable and efficient error indicator for $p$-version finite element method in one dimension with a certain weight. The authors [11] deduced a simple error indicator for spectral Galerkin methods. In [12], the authors investigated LegendreGalerkin spectral method for optimal control problems with integral constraint on state. It is difficult to obtain optimal a posteriori error indicators. Thus, if one gets the constants within upper bound a posteriori error estimates, it is easy to ensure the degree of polynomials to get an acceptable accuracy.

In this paper, we employ Legendre-Galerkin spectral methods to solve optimal control problems with stateconstrained case and calculate constants in upper bound of the a posteriori error indicator, which can be used to decide the least unknowns for acceptable accuracy. With the help of auxiliary systems, we investigate explicit formulae of the constants in the a posteriori error indicator.

The outline of this paper is as follows. In Section 2, the model problem and its Legendre-Galerkin spectral approximations are listed. In Section 3, the constants within the a posteriori error indicator are investigated in detail and the explicit formulae are obtained. The conclusions are given in Section 4. 


\section{A Model Problem and Its Legendre-Galerkin Spectral Approximations}

Throughout this paper we adopt the standard notations of Sobolev spaces [13]. Let $H^{m}(I)$ be a Sobolev space on $I=$ $(-1,1), L^{2}(I)=H^{0}(I)$ and $H_{0}^{1}(I)=\left\{v \in H^{1}(I):\right.$ $v=0$ on $\partial I\}$, and the corresponding norms are denoted by $\|\cdot\|_{m},\|\cdot\|_{0}$, and $\|\cdot\|_{0,1}$, respectively. This work focuses on the Legendre polynomials, which are orthogonal polynomials on $[-1,1]$.

We concern the following distributed convex optimal control problems with integral constraint on state:

$$
(\mathrm{OCP}) \quad\left\{\begin{array}{l}
\min _{y \in K} J(u, y)=\frac{1}{2} \int_{I}\left(y-y_{d}\right)^{2}+\frac{\alpha}{2} \int_{I} u^{2}, \\
\text { s.t. } \quad-y^{\prime \prime}=u \text { in } I, y=0 \text { on } \partial I, y \in K,
\end{array}\right.
$$

where $u \in U=L^{2}(I)$ is the control variable, $y \in K=\{w$ : $\left.\int_{I} w \geq \gamma\right\} \subset H_{0}^{1}(I) \triangleq V$ is the state, and $y_{d} \in L^{2}(I)$ is the observation.

In order to assure the existence and regularity of the solution, we assume that $\alpha$ is a given positive constant and $y_{d}$ is an infinitely smooth function. It is well known that the problem (OCP) has a unique solution (see [3]).

We give some basic notations which will be used in the sequel. Let

$$
\begin{gathered}
(v, w)=\int_{I} v w, \quad \forall v, w \in L^{2}(I), \\
a(v, w)=\int_{I} v^{\prime} w^{\prime}, \quad \forall v, w \in H_{0}^{1}(I) .
\end{gathered}
$$

Hence, the state equation reduces to

$$
a(y, w)=(u, w), \quad \forall w \in H_{0}^{1}(I) .
$$

Then (OCP) can be rewritten as finding $(u, y)$ such that

$$
(\mathscr{P}) \quad\left\{\begin{array}{rl}
\min _{y \in K} & J(u, y)=\frac{1}{2} \int_{I}\left(y-y_{d}\right)^{2}+\frac{\alpha}{2} \int_{I} u^{2} \\
\text { s.t. } & a(y(u), w)=(u, w), \quad \forall w \in V .
\end{array}\right.
$$

We recall the following optimal conditions of $(\mathscr{P})$ (for details please refer to [7]).

Lemma 1. The pair $(u, y) \in U \times V$ is the optimal solution of $(\mathscr{P})$ if and only if there exists a unique pair $(p, \lambda) \in V \times$ $\mathbb{R}_{-}^{1}\left(\mathbb{R}_{-}^{1} \triangleq\left\{c \in \mathbb{R}^{1} ; c \leq 0\right\}\right)$ such that

$$
(O C P-O P T) \quad\left\{\begin{array}{l}
a(y, w)=(u, w), \quad \forall w \in V \\
a(q, p) \\
\quad=\left(y-y_{d}, q\right)+\lambda(1, q), \quad \forall q \in V \\
\lambda(w-y) \leq 0, \quad \forall w \in K, \\
p+\alpha u=0 .
\end{array}\right.
$$

Let $\mathscr{P}_{N}(I)=$ polynomials of degree $\leq N$ on $\left.I\right\}$ and let $V_{N}=\mathscr{P}_{N} \cap H_{0}^{1}(I)$. One prefers to choose appropriate bases of $V_{N}$ such that the resulting linear system is as simple as possible. We denote by $\left\{L_{j}\right\}_{j=0}^{N}$ the Legendre polynomial and employ the following basis functions (see [14]):

$$
\begin{gathered}
U_{N}=\operatorname{span}\left\{L_{0}(x), L_{1}(x), \ldots, L_{N}(x)\right\}, \\
V_{N}=\operatorname{span}\left\{\phi_{0}(x), \phi_{1}(x), \ldots, \phi_{N-2}(x)\right\},
\end{gathered}
$$

where

$$
\phi_{i}(x)=c_{i}\left(L_{i}(x)-L_{i+2}(x)\right), \quad c_{i}=\frac{1}{\sqrt{4 i+6}} .
$$

For $1 \leq j, k \leq N-2$, we denote $a_{j k}=a\left(\phi_{k}(x), \phi_{j}(x)\right)$ and $b_{j k}=\left(\phi_{k}(x), \phi_{j}(x)\right)$. By simple calculations, these coefficients satisfy

$$
\begin{gathered}
a_{j k}= \begin{cases}1, & k=j, \\
0, & k \neq j,\end{cases} \\
b_{j k}=b_{k j}= \begin{cases}c_{k} c_{j}\left(\frac{2}{2 j+1}+\frac{2}{2 j+5}\right), & k=j, \\
-c_{k} c_{j} \frac{2}{2 k+1}, & k=j+2, \\
0, & \text { otherwise. }\end{cases}
\end{gathered}
$$

Then Legendre-Galerkin spectral approximations of (OCP) can be read as finding $\left(u_{N}, y_{N}\right)$ such that

$$
\left(\mathscr{P}^{N}\right)\left\{\begin{array}{l}
\min _{y_{N} \in K} J\left(u_{N}, y_{N}\right)=\frac{1}{2} \int_{I}\left(y_{N}-y_{d}\right)^{2}+\frac{\alpha}{2} \int_{I} u_{N}^{2}, \\
\text { s.t. } a\left(y_{N}, w_{N}\right)=\left(u_{N}, w_{N}\right), \quad \forall w_{N} \in V_{N} .
\end{array}\right.
$$

The Legendre-Galerkin spectral approximations of (5) can be read as follows.

Theorem 2. The pair $\left(u_{N}, y_{N}\right) \in U_{N} \times V_{N}$ is the optimal solution of $\left(\mathscr{P}^{N}\right)$ if and only if there exists a unique pair $\left(p_{N}, \lambda_{N}\right) \in V_{N} \times \mathbb{R}_{-}^{1}$ such that

$$
(O C P-O P T)^{N} \quad\left\{\begin{array}{l}
a\left(y_{N}, v_{N}\right) \\
=\left(u_{N}, v_{N}\right), \quad \forall v_{N} \in V_{N} \\
a\left(q_{N}, p_{N}\right) \\
\quad=\left(y_{N}-y_{d}, q_{N}\right) \\
\quad+\lambda_{N}\left(1, q_{N}\right), \quad \forall q_{N} \in V_{N} \\
\lambda_{N}\left(1, w_{N}-y_{N}\right) \leq 0, \quad \forall w_{N} \in K_{N} \\
\alpha u_{N}+p_{N}=0 .
\end{array}\right.
$$

\section{Constants within the A Posteriori Error Estimates}

In this section, we calculate all constants within the a posteriori error estimates. Here, we analyze the constant in the Poincaré inequality.

For all $v \in W_{0}^{1, p}(I), 1 \leq p<\infty$, we recall the Poincaré inequality with $L^{2}$-norm as (see [15])

$$
\|v\|_{0} \leq \frac{|I|}{2}\left\|v^{\prime}\right\|_{0} .
$$


Now, we are at the point to investigate all constants in detail. We introduce an auxiliary state $y\left(u_{N}\right) \in H_{0}^{1}(I)$, which satisfies

$$
a\left(y\left(u_{N}\right), w\right)=\left(u_{N}, w\right), \quad \forall w \in H_{0}^{1}(I) .
$$

Subtracting (12) from (3), we get

$$
a\left(y-y\left(u_{N}\right), w\right)=\left(u-u_{N}, w\right), \quad \forall w \in H_{0}^{1}(I) .
$$

Let $w=y\left(u_{N}\right)-y \in H_{0}^{1}(I)$. It is clear that

$$
a\left(y\left(u_{N}\right)-y, y\left(u_{N}\right)-y\right)=\left(u_{N}-u, y\left(u_{N}\right)-y\right) .
$$

Then

$$
\begin{aligned}
\left\|\left(y\left(u_{N}\right)-y\right)^{\prime}\right\|_{0}^{2} & \leq\left\|u_{N}-u\right\|_{0}\left\|y\left(u_{N}\right)-y\right\|_{0} \\
& \leq \frac{|I|}{2}\left\|u_{N}-u\right\|_{0}\left\|\left(y\left(u_{N}\right)-y\right)^{\prime}\right\|_{0},
\end{aligned}
$$

which means

$$
\left\|\left(y\left(u_{N}\right)-y\right)^{\prime}\right\|_{0} \leq \frac{|I|}{2}\left\|u_{N}-u\right\|_{0} .
$$

Hence

$$
\begin{aligned}
\| y & \left(u_{N}\right)-y \|_{1} \\
& \leq\left(\left\|\left(y\left(u_{N}\right)-y\right)^{\prime}\right\|_{0}^{2}+\left(\frac{|I|}{2}\right)^{2}\left\|\left(y\left(u_{N}\right)-y\right)^{\prime}\right\|_{0}^{2}\right)^{1 / 2} \\
& =\left(1+\left(\frac{|I|}{2}\right)^{2}\right)^{1 / 2}\left\|\left(y\left(u_{N}\right)-y\right)^{\prime}\right\|_{0} .
\end{aligned}
$$

Then

$$
\left\|y\left(u_{N}\right)-y\right\|_{1} \leq\left(1+\left(\frac{|I|}{2}\right)^{2}\right)^{1 / 2} \frac{|I|}{2}\left\|u_{N}-u\right\|_{0} .
$$

We denote by $c_{1}$ the constant in (18); that is,

$$
c_{1}=\left(1+\left(\frac{|I|}{2}\right)^{2}\right)^{1 / 2} \frac{|I|}{2} .
$$

Similarly, we introduce an auxiliary state $p\left(u_{N}\right) \in H^{1}(I)$, which satisfies

$$
a\left(q, p\left(u_{N}\right)\right)=\left(y\left(u_{N}\right)-y_{d}, q\right)+\lambda(1, q), \quad \forall q \in H_{0}^{1}(I) .
$$

Subtracting (20) from the continuous systems (5), we get

$$
\begin{array}{r}
a\left(p-p\left(u_{N}\right), w\right) \\
=\left(y-y\left(u_{N}\right), w\right)+\left(\lambda-\lambda_{N}\right)(1, w), \\
\forall w \in H_{0}^{1}(I) .
\end{array}
$$

We select $\varphi \in C_{0}^{\infty}(I)$ which satisfies $\bar{\varphi}=1$, where $\bar{\varphi} \triangleq$ $\int_{I} \varphi /|I|=1$ denotes the integral average on $I$ of the function $\varphi$ and $\|\varphi\|_{1} \leq C_{\varphi}$. Obviously, $\overline{p-p\left(u_{N}\right)} \varphi \in C_{0}^{\infty}(I)$. In fact, $p-p\left(u_{N}\right)-\overline{p-p\left(u_{N}\right)} \varphi \in H_{0}^{1}(I)$. Then there hold

$$
\begin{aligned}
\left\|p-p\left(u_{N}\right)\right\|_{1}^{2} & \left(1+\left(\frac{|I|}{2}\right)^{2}\right)\left|p-p\left(u_{N}\right)\right|_{1}^{2} \\
\leq & \left(1+\left(\frac{|I|}{2}\right)^{2}\right) a\left(p-p\left(u_{N}\right), p-p\left(u_{N}\right)\right) \\
= & \left(1+\left(\frac{|I|}{2}\right)^{2}\right) \\
& \quad \times\left\{a\left(\overline{p-p\left(u_{N}\right)} \varphi, p-p\left(u_{N}\right)\right)\right. \\
& \left.+\left(y-y\left(u_{N}\right), p-p\left(u_{N}\right)-\overline{p-p\left(u_{N}\right)} \varphi\right)\right\}
\end{aligned}
$$

$$
\begin{aligned}
\leq & \left(1+\left(\frac{|I|}{2}\right)^{2}\right) \\
& \times\left\{\left|\overline{p-p\left(u_{N}\right)}\right| \cdot\left(\frac{\epsilon_{1}}{2}|\varphi|_{1}^{2}+\frac{1}{2 \epsilon_{1}}\left|p-p\left(u_{N}\right)\right|_{1}^{2}\right)\right.
\end{aligned}
$$$$
+\frac{\epsilon_{2}}{2}\left\|y-y\left(u_{N}\right)\right\|_{0}^{2}
$$$$
\left.+\frac{1}{\epsilon_{2}}\left(\left\|p-p\left(u_{N}\right)\right\|_{0}^{2}+\left|\overline{p-p\left(u_{N}\right)}\right|^{2} \cdot\|\varphi\|_{0}^{2}\right)\right\}
$$

where we used the generalized Schwarz inequality, continuous systems (5), and auxiliary equation (20). Let

$$
\left(1+\left(\frac{|I|}{2}\right)^{2}\right) \frac{\left|\overline{p-p\left(u_{N}\right)}\right|}{2 \epsilon_{1}}=\left(1+\left(\frac{|I|}{2}\right)^{2}\right) \frac{1}{\epsilon_{2}}=\frac{1}{2} .
$$

Then

$$
\epsilon_{1}=\left(1+\left(\frac{|I|}{2}\right)^{2}\right)\left|\overline{p-p\left(u_{N}\right)}\right|, \quad \epsilon_{2}=2\left(1+\left(\frac{|I|}{2}\right)^{2}\right) .
$$


It is clear that (22) reduces to

$$
\begin{aligned}
& \left\|p-p\left(u_{N}\right)\right\|_{1}^{2} \\
& \leq\left(1+\left(\frac{|I|}{2}\right)^{2}\right) \\
& \times\left\{\frac{1+(|I| / 2)^{2}}{2}\left|\overline{p-p\left(u_{N}\right)}\right|^{2} \cdot|\varphi|_{1}^{2}\right. \\
& +\frac{\left|\overline{p-p\left(u_{N}\right)}\right|^{2}}{2\left(1+(|I| / 2)^{2}\right)}\|\varphi\|_{0}^{2} \\
& \left.+\left(1+\left(\frac{|I|}{2}\right)^{2}\right)\left\|y-y\left(u_{N}\right)\right\|_{0}^{2}\right\} \\
& +\frac{1}{2}\left\|p-p\left(u_{N}\right)\right\|_{1}^{2} \text {. }
\end{aligned}
$$

Then

$$
\begin{aligned}
\| p- & p\left(u_{N}\right) \|_{1}^{2} \\
\leq & 2\left(1+\left(\frac{|I|}{2}\right)^{2}\right) \\
& \times\left\{\left(1+\left(\frac{|I|}{2}\right)^{2}\right)\left|\overline{p-p\left(u_{N}\right)}\right|^{2} \cdot\|\varphi\|_{1}^{2}\right. \\
& \left.+\left(1+\left(\frac{|I|}{2}\right)^{2}\right)\left\|y-y\left(u_{N}\right)\right\|_{0}^{2}\right\} \\
= & 2\left(1+\left(\frac{|I|}{2}\right)^{2}\right)^{2}\left\{\mid \frac{p-p\left(u_{N}\right)}{2} \cdot\|\varphi\|_{1}^{2}\right. \\
& \left.+\left\|y-y\left(u_{N}\right)\right\|_{0}^{2}\right\},
\end{aligned}
$$

where we used $\left(1+(|I| / 2)^{2}\right)\left|\overline{p-p\left(u_{N}\right)}\right|^{2} \geq(1 /(1+(|I| /$ $\left.\left.2)^{2}\right)\right)\left|\overline{p-p\left(u_{N}\right)}\right|^{2}$.

Hence

$$
\begin{aligned}
\| p & -p\left(u_{N}\right) \|_{1}^{2} \\
& \leq 2\left(1+\left(\frac{|I|}{2}\right)^{2}\right)^{2}
\end{aligned}
$$

$$
\begin{aligned}
\times & \left\{\|\varphi\|_{1}^{2} \cdot \frac{1}{|I|}\left(\alpha\left\|u-u_{N}\right\|_{0}+\left\|p_{N}-p\left(u_{N}\right)\right\|_{0}\right)^{2}\right. \\
& \left.+\left(1+\left(\frac{|I|}{2}\right)^{2}\right)\left(\frac{|I|}{2}\right)^{2}\left\|u-u_{N}\right\|_{0}^{2}\right\} \\
= & 2\left(1+\left(\frac{|I|}{2}\right)^{2}\right)^{2} \\
& \times\left\{\left[\frac{2\|\varphi\|_{1}^{2} \alpha^{2}}{|I|}+\left(1+\left(\frac{|I|}{2}\right)^{2}\right)\left(\frac{|I|}{2}\right)^{2}\right]\right. \\
& \left.\times\left\|u-u_{N}\right\|_{0}^{2}+\frac{2\|\varphi\|_{1}^{2}}{|I|}\left\|p_{N}-p\left(u_{N}\right)\right\|_{0}^{2}\right\} \\
= & 2\left(1+\left(\frac{|I|}{2}\right)^{2}\right)^{2} \max \left\{\frac{2\|\varphi\|_{1}^{2} \alpha^{2}}{|I|}+c_{1}^{2}, \frac{2\|\varphi\|_{1}^{2}}{|I|}\right\} \\
& \times\left\{\left\|u-u_{N}\right\|_{0}^{2}+\left\|p_{N}-p\left(u_{N}\right)\right\|_{0}^{2}\right\},
\end{aligned}
$$

which means that

$$
\begin{aligned}
& \left\|p-p\left(u_{N}\right)\right\|_{1} \\
& \leq\left(2\left(1+\left(\frac{|I|}{2}\right)^{2}\right)^{2}\right. \\
& \left.\quad \times \max \left\{\frac{2 C_{\varphi}^{2} \alpha^{2}}{|I|}+\left(1+\left(\frac{|I|}{2}\right)^{2}\right)\left(\frac{|I|}{2}\right)^{2}, \frac{2 C_{\varphi}^{2}}{|I|}\right\}\right)^{1 / 2} \\
& \quad \times\left\{\left\|u-u_{N}\right\|_{0}+\left\|p_{N}-p\left(u_{N}\right)\right\|_{0}\right\} .
\end{aligned}
$$

Denote by $c_{2}$ the constant in (28). With simple calculation, we have

$$
c_{2}=\sqrt{2\left(1+\left(\frac{|I|}{2}\right)^{2}\right)^{2} \max \left\{\frac{2 C_{\varphi}^{2} \alpha^{2}}{|I|}+c_{1}^{2}, \frac{2 C_{\varphi}^{2}}{|I|}\right\}}
$$

We select $\varphi \in C_{0}^{\infty}(I)$ which satisfies $\bar{\varphi}=1$ and $\|\varphi\|_{1} \leq C_{\varphi}$. For instance, $\varphi=(3 / 2)\left(1-x^{2}\right)$, which satisfies

$$
\|\varphi\|_{1}=\frac{3}{2} \sqrt{\frac{74}{15}} \triangleq C_{\varphi} .
$$

Meanwhile,

$$
\begin{aligned}
& \left(\lambda-\lambda_{N},\left(\lambda-\lambda_{N}\right) \varphi\right) \\
& \quad=\left(\lambda-\lambda_{N}\right)^{2} \int_{I} \varphi=\left(\lambda-\lambda_{N}\right)^{2}|I|=\left\|\lambda-\lambda_{N}\right\|_{0}^{2} .
\end{aligned}
$$


Hence

$$
\begin{aligned}
\| \lambda- & \lambda_{N} \|_{0}^{2} \\
= & \left(\lambda-\lambda_{N},\left(\lambda-\lambda_{N}\right) \varphi\right) \\
= & a\left(\left(\lambda-\lambda_{N}\right) \varphi, p-p\left(u_{N}\right)\right) \\
& \quad-\left(y-y\left(u_{N}\right),\left(\lambda-\lambda_{N}\right) \varphi\right) \\
\leq & \left|\lambda-\lambda_{N}\right|\left\{\left\|\varphi^{\prime}\right\|_{0} \cdot\left\|\left(p-p\left(u_{N}\right)\right)^{\prime}\right\|_{0}\right. \\
& \left.+\|\varphi\|_{0} \cdot\left\|y-y\left(u_{N}\right)\right\|_{0}\right\} \\
\leq & \frac{\epsilon_{1}}{2}\left|\lambda-\lambda_{N}\right|^{2} \cdot\left\|\varphi^{\prime}\right\|_{0}^{2}+\frac{1}{2 \epsilon_{1}}\left\|\left(p-p\left(u_{N}\right)\right)^{\prime}\right\|_{0}^{2} \\
& +\frac{\epsilon_{2}}{2}\left|\lambda-\lambda_{N}\right|^{2} \cdot\|\varphi\|_{0}^{2}+\frac{1}{2 \epsilon_{2}}\left\|y-y\left(u_{N}\right)\right\|_{0}^{2} \\
= & \frac{\epsilon_{1}}{2|I|}\left\|\lambda-\lambda_{N}\right\|_{0}^{2}\|\varphi\|_{1}^{2} \\
& +\frac{1}{2 \epsilon_{1}}\left(\left\|\left(p-p\left(u_{N}\right)\right)^{\prime}\right\|_{0}^{2}+\left\|y-y\left(u_{N}\right)\right\|_{0}^{2}\right),
\end{aligned}
$$

where $\epsilon_{1}=\epsilon_{2}=|I| /\|\varphi\|_{1}^{2}$.

Thus

$$
\begin{aligned}
\left\|\lambda-\lambda_{N}\right\|_{0}^{2} & \leq \frac{\|\varphi\|_{1}^{2}}{|I|}\left\{\left\|\left(p-p\left(u_{N}\right)\right)^{\prime}\right\|_{0}^{2}+\left\|y-y\left(u_{N}\right)\right\|_{0}^{2}\right\} \\
& \leq \frac{\|\varphi\|_{1}^{2}}{|I|}\left\{\left\|p-p\left(u_{N}\right)\right\|_{1}^{2}+\left\|y-y\left(u_{N}\right)\right\|_{0}^{2}\right\} .
\end{aligned}
$$

With the constant $c_{2}$, we infer that

$$
\begin{aligned}
\| \lambda- & \lambda_{N} \|_{0}^{2} \\
\leq & \frac{C_{\varphi}^{2}}{|I|}\left\{4\left(1+\left(\frac{|I|}{2}\right)^{2}\right)^{2}\right. \\
& \quad \times \max \left\{\frac{2 C_{\varphi}^{2} \alpha^{2}}{|I|}+\left(1+\left(\frac{|I|}{2}\right)^{2}\right)\left(\frac{|I|}{2}\right)^{2}, \frac{2 C_{\varphi}^{2}}{|I|}\right\} \\
& \left.+\left(1+\left(\frac{|I|}{2}\right)^{2}\right)\left(\frac{|I|}{2}\right)^{2}\right\} \\
& \times\left\{\left\|p_{N}-p\left(u_{N}\right)\right\|_{0}^{2}+\left\|u-u_{N}\right\|_{0}^{2}\right\} \\
= & \frac{C_{\varphi}^{2}}{|I|}\left\{2 c_{2}^{2}+c_{1}^{2}\right\}\left\{\left\|p_{N}-p\left(u_{N}\right)\right\|_{0}^{2}+\left\|u-u_{N}\right\|_{0}^{2}\right\} .
\end{aligned}
$$

Then

$$
\begin{aligned}
\| \lambda & -\lambda_{N} \|_{0} \\
& \leq \frac{C_{\varphi}^{2}}{|I|}\left\{2 c_{2}^{2}+c_{1}^{2}\right\}\left\{\left\|p_{N}-p\left(u_{N}\right)\right\|_{0}+\left\|u-u_{N}\right\|_{0}\right\} .
\end{aligned}
$$

We denote by $c_{3}$ the constant in (35); that is,

$$
c_{3}=\sqrt{\frac{C_{\varphi}^{2}}{|I|}\left\{2 c_{2}^{2}+c_{1}^{2}\right\}} .
$$

We calculate the error of $u$ in $L^{2}$-norm as follows:

$$
\begin{aligned}
\| u- & u_{N} \|_{0}^{2} \\
\leq & \left(p_{N}-p\left(u_{N}\right), u-u_{N}\right) \\
& -\left(\lambda-\lambda_{N}, y_{N}-y\left(u_{N}\right)\right) \\
\leq & \frac{\epsilon_{1}}{2}\left\|p_{N}-p\left(u_{N}\right)\right\|_{0}^{2}+\frac{1}{2 \epsilon_{1}}\left\|u-u_{N}\right\|_{0}^{2} \\
& +\frac{1}{2 \epsilon_{2}}\left\|y_{N}-y\left(u_{N}\right)\right\|_{0}^{2} \\
& +\epsilon_{2} \cdot c_{3}^{2}\left\{\left\|u-u_{N}\right\|_{0}^{2}+\left\|p_{N}-p\left(u_{N}\right)\right\|_{0}^{2}\right\} \\
= & \left(\frac{\epsilon_{1}}{2}+\epsilon_{2} \cdot c_{3}^{2}\right)\left\|p_{N}-p\left(u_{N}\right)\right\|_{0}^{2} \\
& +\left(\frac{1}{2 \epsilon_{1}}+\epsilon_{2} \cdot c_{3}^{2}\right)\left\|u-u_{N}\right\|_{0}^{2} \\
& +\frac{1}{2 \epsilon_{2}}\left\|y_{N}-y\left(u_{N}\right)\right\|_{0}^{2} .
\end{aligned}
$$

Provided that $1 / 2 \epsilon_{1}+\epsilon_{2} \cdot c_{3}^{2}=1 / 2$, we get

$$
\begin{aligned}
\| u- & u_{N} \|_{0}^{2} \\
\leq & 2\left(\frac{\epsilon_{1}}{2}+\epsilon_{2} \cdot c_{3}^{2}\right)\left\|p_{N}-p\left(u_{N}\right)\right\|_{0}^{2} \\
& \quad+\frac{1}{\epsilon_{2}}\left\|y_{N}-y\left(u_{N}\right)\right\|_{0}^{2} \\
= & \max \left\{\epsilon_{1}+2 \epsilon_{2} \cdot c_{3}^{2}, \frac{1}{\epsilon_{2}}\right\}\left\{\left\|p_{N}-p\left(u_{N}\right)\right\|_{0}^{2}\right. \\
& \left.+\left\|y_{N}-y\left(u_{N}\right)\right\|_{0}^{2}\right\} .
\end{aligned}
$$

Considering the item $\max \left\{\epsilon_{1}+2 \epsilon_{2} \cdot c_{3}^{2}, 1 / \epsilon_{2}\right\}$ with the constraint $1 / 2 \epsilon_{1}+\epsilon_{2} \cdot c_{3}^{2}=1 / 2$, we get

$$
F\left(\epsilon_{1}\right)=\max \left\{\epsilon_{1}+1-\frac{1}{\epsilon_{1}}, \frac{2 c_{3}^{2} \epsilon_{1}}{\epsilon_{1}-1}\right\} .
$$

In fact, for $\forall \epsilon_{1}>1$, the derivation of the following function

$$
f\left(\epsilon_{1}\right)=\epsilon_{1}+1-\frac{1}{\epsilon_{1}}-\frac{2 c_{3}^{2} \epsilon_{1}}{\epsilon_{1}-1}
$$

is

$$
f^{\prime}\left(\epsilon_{1}\right)=1+\frac{1}{\epsilon_{1}^{2}}+\frac{2 c_{3}^{2}}{\left(\epsilon_{1}-1\right)^{2}} \geq 0 .
$$


Then we have $\epsilon_{1}^{0}=2 c_{3}^{2}+\epsilon>1$ and

$$
\lim _{\epsilon \rightarrow 0} f\left(\epsilon_{1}^{0}\right)=0 \text {. }
$$

Now, we are at the point to investigate

$$
\min F\left(\epsilon_{1}\right) \text {. }
$$

If $\epsilon_{1}=\epsilon_{1}^{0}$, we get

$$
\min F\left(\epsilon_{1}\right)=\epsilon_{1}^{0}+1-\frac{1}{\epsilon_{1}^{0}} \leq 2 c_{3}^{2}+\epsilon+1 .
$$

If $1<\epsilon_{1}<\epsilon_{1}^{0}, f\left(\epsilon_{1}\right)<0$, we obtain

$$
F\left(\epsilon_{1}\right)=\frac{2 c_{3}^{2} \epsilon_{1}}{\epsilon_{1}-1}, \quad F^{\prime}\left(\epsilon_{1}\right)=-\frac{2 c_{3}^{2}}{\left(\epsilon_{1}-1\right)^{2}}<0 .
$$

Then

$$
\lim _{\epsilon^{\prime} \rightarrow 0} \min F\left(\epsilon_{1}\right)=2 c_{3}^{2}\left(1+\frac{1}{2 c_{3}^{2}+\epsilon-\epsilon^{\prime}-1}\right)<4 c_{3}^{2} .
$$

If $\epsilon_{1}>\epsilon_{1}^{0}, f\left(\epsilon_{1}\right)>0$, we infer that

$$
F\left(\epsilon_{1}\right)=\epsilon_{1}+1-\frac{1}{\epsilon_{1}}, \quad F^{\prime}\left(\epsilon_{1}\right)=1+\frac{1}{\epsilon_{1}^{2}}>0 .
$$

Then there hold

$$
\lim _{\epsilon^{\prime \prime} \rightarrow 0} \min F\left(\epsilon_{1}\right)=2 c_{3}^{2}+\epsilon+\epsilon^{\prime \prime}+1-\frac{1}{2 c_{3}^{2}+\epsilon+\epsilon^{\prime \prime}}<2 c_{3}^{2}+1 .
$$

Combining the above discussions, we deduce that

$$
\min \left\{\max \left\{\epsilon_{1}+2 \epsilon_{2} \cdot c_{3}^{2}, \frac{1}{\epsilon_{2}}\right\}\right\}<2 c_{3}^{2}+1
$$

Obviously,

$$
\begin{aligned}
\| u & -u_{N} \|_{0} \\
\leq & \sqrt{2 c_{3}^{2}+1}\left\{\left\|p_{N}-p\left(u_{N}\right)\right\|_{0}+\left\|y_{N}-y\left(u_{N}\right)\right\|_{0}\right\} .
\end{aligned}
$$

We denote by $c_{4}$ the constant in (50); that is,

$$
c_{4}=\sqrt{2 c_{3}^{2}+1} \text {. }
$$

For any $v \in L^{2}(I)$, we define a projection operator $\mathbb{P}_{N}$ : $L^{2}(I) \rightarrow V_{N}$, which satisfies

$$
\left(\mathbb{P}_{N} v-v, w_{N}\right)=0, \quad \forall w_{N} \in U_{N} .
$$

Lemma 3. For all $v \in H^{\sigma}(I)(\sigma \geq 0)$, one has

$$
\left\|\mathbb{P}_{N} v-v\right\|_{0} \leq c_{5} N^{-\sigma}\|v\|_{\sigma}
$$

where $c_{5}=2 \sqrt{2}$.
Proof. Firstly, assuming that $\sigma=2 p(p \geq 1)$ is integer, we define a differential operator as

$$
A=\frac{d}{d x}\left(\left(1-x^{2}\right) \frac{d}{d x}\right) .
$$

From the fact that

$$
\frac{d}{d x}\left(\left(1-x^{2}\right) \frac{d L_{k}}{d x}\right)+k(k+1) L_{k}=0,
$$

it is easy to get

$$
\begin{aligned}
\widehat{v}_{k} & =\left(k+\frac{1}{2}\right)\left(v, L_{k}\right) \\
& =\frac{k+1 / 2}{k(k+1)} \int_{-1}^{1} A L_{k}(x) v(x) d x \\
& =-\frac{k+1 / 2}{k(k+1)} \int_{-1}^{1} A v(x) L_{k}(x) d x \\
& =-\frac{k+1 / 2}{k(k+1)}\left(A v(x), L_{k}(x)\right) .
\end{aligned}
$$

By iterations, we obtain

$$
\widehat{v}_{k}=\left(\frac{-1}{k(k+1)}\right)^{p}\left(k+\frac{1}{2}\right)\left(A^{p} v, L_{k}\right) .
$$

Secondly, for all $v \in H^{2 p}(I)$, we note that $A^{p} v=\sum_{i=0}^{\infty} \alpha_{i} L_{i}(x)$ and

$$
\begin{gathered}
\left(A^{p} v(x), L_{k}(x)\right)=\alpha_{i}\left(k+\frac{1}{2}\right)^{-1}, \\
\left\|A^{p} v\right\|_{0}^{2}=\sum_{k=0}^{\infty}\left|\alpha_{k}\right|^{2}\left(k+\frac{1}{2}\right)^{-1} .
\end{gathered}
$$

Hence

$$
\begin{aligned}
\left\|\mathbb{P}_{N} v-v\right\|_{0}^{2} & =\sum_{k=N+1}^{\infty}\left(k+\frac{1}{2}\right)^{-1}\left|\widehat{v}_{k}\right|^{2} \\
& =\sum_{k=N+1}^{\infty}\left(\frac{1}{k(k+1)}\right)^{2 p}\left(k+\frac{1}{2}\right)\left|A^{p} v, L_{k}\right|^{2} \\
& \leq N^{-4 p} \sum_{k=N+1}^{\infty}\left(k+\frac{1}{2}\right)\left|\alpha_{k}\right|^{2}\left(k+\frac{1}{2}\right)^{-2} \\
& \leq N^{-4 p}\left\|A^{p} v\right\|_{0}^{2} .
\end{aligned}
$$

Finally, there hold

$$
\begin{aligned}
|A v|^{2} & =\left|\frac{d}{d x}\left(\left(1-x^{2}\right) \frac{d v}{d x}\right)\right|^{2} \\
& =\left|\left(1-x^{2}\right) v^{\prime \prime}-2 x v^{\prime}\right|^{2} \\
& \leq\left(\left|v^{\prime \prime}\right|+2\left|v^{\prime}\right|\right)^{2} \\
& \leq 2 v^{\prime \prime 2}+8 v^{\prime 2} \\
& \leq 8\left\{v^{\prime \prime 2}+v^{\prime 2}+v^{2}\right\},
\end{aligned}
$$


which means that

$$
\|A v\|_{0}^{2} \leq 8\|v\|_{2}^{2}
$$

Let $p=1, \sigma=2$. It is clear that

$$
\left\|\mathbb{P}_{N} v-v\right\|_{0} \leq \sqrt{8} N^{-2}\|v\|_{2}
$$

This completes the proof.

Now, we are at the point to calculate the constant for $\left\|y_{N}-y\left(u_{N}\right)\right\|_{1}+\left\|p_{N}-p\left(u_{N}\right)\right\|_{1}$. Similarly, let $E^{p}=p_{N}-$ $p\left(u_{N}\right)$ and let $E_{I}^{p}=\mathbb{P}_{N} E^{p} \in V_{N}$. Then

$$
\begin{aligned}
&\left\|p_{N}-p\left(u_{N}\right)\right\|_{1}^{2}\left(1+\left(\frac{|I|}{2}\right)^{2}\right)\left(a\left(E^{p}, E^{p}-E_{I}^{p}\right)+\left(y\left(u_{N}\right)-y_{N}, E_{I}^{p}\right)\right) \\
&=\left(1+\left(\frac{|I|}{2}\right)^{2}\right)\left(a\left(p\left(u_{N}\right)-p_{N}, E^{p}-E_{I}^{p}\right)\right. \\
&\left.+\left(y\left(u_{N}\right)-y_{N}, E_{I}^{p}\right)\right) \\
&=\left(1+\left(\frac{|I|}{2}\right)^{2}\right)+\left(\left(-p^{\prime \prime}\left(u_{N}\right), E^{p}-E_{I}^{p}\right)\right. \\
&\left.+\left(p_{N}^{\prime \prime}, E^{p}-E_{I}^{p}\right)+\left(y\left(u_{N}\right)-y_{N}, E_{I}^{p}\right)\right) \\
&=\left(1+\left(\frac{|I|}{2}\right)^{2}\right)\left(\left(y_{N}-y_{d}+\lambda_{N}+p_{N}^{\prime \prime}, E^{p}-E_{I}^{p}\right)\right. \\
&\left.+\left(y\left(u_{N}\right)-y_{N}, E^{p}\right)\right) \\
& \leq\left(1+\left(\frac{|I|}{2}\right)^{2}\right)\left\|E^{p}\right\|_{1}\left\{c_{5} N^{-1}\left\|y_{N}-y_{d}+\lambda_{N}+p_{N}^{\prime \prime}\right\|_{0}\right. \\
&\left.+\left\|y_{N}-y\left(u_{N}\right)\right\|_{0}\right\},
\end{aligned}
$$

which means that

$$
\begin{gathered}
\left\|p_{N}-p\left(u_{N}\right)\right\|_{1} \\
\leq\left(1+\left(\frac{|I|}{2}\right)^{2}\right)\left\{c_{5} N^{-1}\left\|y_{N}-y_{d}+\lambda_{N}+p_{N}^{\prime \prime}\right\|_{0}\right. \\
\left.+\left\|y_{N}-y\left(u_{N}\right)\right\|_{0}\right\} .
\end{gathered}
$$

Likewise, let $E^{y}=y_{N}-y\left(u_{N}\right)$ and let $E_{I}^{y}=\mathbb{P}_{1, N}^{0} E^{y} \in V_{N}$. Then

$$
\begin{aligned}
\left\|y_{N}-y\left(u_{N}\right)\right\|_{1}^{2} \\
=\left\|E^{y}\right\|_{1}^{2} \leq\left(1+\left(\frac{|I|}{2}\right)^{2}\right) a\left(E^{y}, E^{y}\right) \\
\quad=\left(1+\left(\frac{|I|}{2}\right)^{2}\right) a\left(E^{y}-E_{I}^{y}, E^{y}\right) \\
=\left(1+\left(\frac{|I|}{2}\right)^{2}\right)\left(-\left(u_{N}+y_{N}^{\prime \prime}\right), E^{y}-E_{I}^{y}\right) \\
\quad \leq\left(1+\left(\frac{|I|}{2}\right)^{2}\right) c_{5} N^{-1}\left\|u_{N}+y_{N}^{\prime \prime}\right\|_{0} \cdot\left\|E^{y}\right\|_{1},
\end{aligned}
$$

which is equivalent to

$$
\left\|y_{N}-y\left(u_{N}\right)\right\|_{1} \leq\left(1+\left(\frac{|I|}{2}\right)^{2}\right) c_{5} N^{-1}\left\|u_{N}+y_{N}^{\prime \prime}\right\|_{0} .
$$

Hence

$$
\left\|y_{N}-y\left(u_{N}\right)\right\|_{1}+\left\|p_{N}-p\left(u_{N}\right)\right\|_{1} \leq c_{6} \eta,
$$

where

$$
\begin{gathered}
c_{6}=\left(1+\left(\frac{|I|}{2}\right)^{2}\right)^{2} c_{5}, \\
\eta=N^{-1}\left\|y_{N}-y_{d}+\lambda_{N}+p_{N}^{\prime \prime}\right\|_{0}+N^{-1}\left\|u_{N}+y_{N}^{\prime \prime}\right\|_{0} .
\end{gathered}
$$

Combining the above analyses, we get that

$$
\begin{aligned}
\| u- & u_{N}\left\|_{0}+\right\| y-y_{N}\left\|_{1}+\right\| p-p_{N}\left\|_{1}+\right\| \lambda-\lambda_{N} \|_{0} \\
\leq & \left\|u-u_{N}\right\|_{0}+\left\|y-y\left(u_{N}\right)\right\|_{1}+\left\|y_{N}-y\left(u_{N}\right)\right\|_{1} \\
& +\left\|p-p\left(u_{N}\right)\right\|_{1}+\left\|p_{N}-p\left(u_{N}\right)\right\|_{1}+\left\|\lambda-\lambda_{N}\right\|_{0} \\
\leq & \left\|y_{N}-y\left(u_{N}\right)\right\|_{1}+\left\|p_{N}-p\left(u_{N}\right)\right\|_{1} \\
& +c_{4}\left\{\left\|p_{N}-p\left(u_{N}\right)\right\|_{0}+\left\|y_{N}-y\left(u_{N}\right)\right\|_{0}\right\} \\
& +c_{1} c_{4}\left\{\left\|p_{N}-p\left(u_{N}\right)\right\|_{0}+\left\|y_{N}-y\left(u_{N}\right)\right\|_{0}\right\} \\
& +c_{2}\left\{\left\|p_{N}-p\left(u_{N}\right)\right\|_{0}\right. \\
& \left.\quad+c_{4}\left(\left\|p_{N}-p\left(u_{N}\right)\right\|_{0}+\left\|y_{N}-y\left(u_{N}\right)\right\|_{0}\right)\right\} \\
& +c_{3}\left\{\left\|p_{N}-p\left(u_{N}\right)\right\|_{0, I}\right. \\
& \left.\quad+c_{4}\left(\left\|p_{N}-p\left(u_{N}\right)\right\|_{0}+\left\|y_{N}-y\left(u_{N}\right)\right\|_{0}\right)\right\} \\
\leq & \left\|p_{N}-p\left(u_{N}\right)\right\|_{1}\left\{c_{4}+1+c_{1} c_{4}+c_{2}\left(c_{4}+1\right)+c_{3}\left(c_{4}+1\right)\right\} \\
& +\left\|y_{N}-y\left(u_{N}\right)\right\|_{1}\left\{c_{4}+1+c_{1} c_{4}+c_{2} c_{4}+c_{3} c_{4}\right\} \\
\leq & \left\{c_{4}+1+c_{1} c_{4}+c_{2}\left(c_{4}+1\right)+c_{3}\left(c_{4}+1\right)\right\} \\
& \times\left\{\left\|p_{N}-p\left(u_{N}\right)\right\|_{1}+\left\|y_{N}-y\left(u_{N}\right)\right\|_{1}\right\} \\
\leq & \left\{c_{4}+1+c_{1} c_{4}+c_{2}\left(c_{4}+1\right)+c_{3}\left(c_{4}+1\right)\right\} c_{6} \eta
\end{aligned}
$$


which means that

$$
\left\|u-u_{N}\right\|_{0}+\left\|y-y_{N}\right\|_{1}+\left\|p-p_{N}\right\|_{1}+\left\|\lambda-\lambda_{N}\right\|_{0} \leq C \eta
$$

where

$$
\begin{aligned}
& C=\left\{1+c_{4}+c_{1} c_{4}+c_{2}\left(c_{4}+1\right)+c_{3}\left(c_{4}+1\right)\right\} c_{6}, \\
& c_{1}=\left(1+\left(\frac{|I|}{2}\right)^{2}\right)^{1 / 2} \frac{|I|}{2}, \\
& c_{2}=\sqrt{2\left(1+\left(\frac{|I|}{2}\right)^{2}\right)^{2} \max \left\{\frac{2 C_{\varphi}^{2} \alpha^{2}}{|I|}+c_{1}^{2}, \frac{2 C_{\varphi}^{2}}{|I|}\right\},} \\
& c_{3}=\sqrt{\frac{C_{\varphi}^{2}}{|I|}\left\{2 c_{2}^{2}+c_{1}^{2}\right\}}, \\
& c_{4}=\sqrt{2 c_{3}^{2}+1}, \\
& c_{5}=\sqrt{2}, \\
& c_{6}=\left(1+\left(\frac{|I|}{2}\right)^{2}\right)^{2} c_{5} .
\end{aligned}
$$

\section{Conclusions}

This paper discusses the explicit formulae of constants within upper bound of the a posteriori error estimate for optimal control problems with Legendre-Galerkin spectral methods in one dimension. Thus, with those formulae, it is easy to choose a suitable degree of polynomials to obtain an acceptable accuracy. In the future, we will study the corresponding constants in lower bound of the a posteriori error indicator. Meanwhile, the corresponding constants in a twodimensional domain will be investigated.

\section{Conflict of Interests}

The author declares that there is no conflict of interests regarding the publication of this paper.

\section{Acknowledgments}

This work is partially supported by National Natural Science Foundation of China (no. 11201212), Promotive Research Fund for Excellent Young and Middle-aged Scientists of Shandong Province (no. BS2012DX004), AMEP, and the Special Funds for Doctoral Authorities of Linyi University.

\section{References}

[1] D. Gottlieb and S. A. Orszag, Numerical Analysis of Spectral Methods: Theory and Applications, Society for Industrial and Applied Mathematics, Philadelphia, Pa, USA, 1977.
[2] R. Li, W. Liu, H. Ma, and T. Tang, "Adaptive finite element approximation for distributed elliptic optimal control problems," SIAM Journal on Control and Optimization, vol. 41, no. 5, pp. 1321-1349, 2002.

[3] W. B. Liu and N. N. Yan, "A posteriori error estimates for distributed convex optimal control problems," Advances in Computational Mathematics, vol. 15, no. 1-4, pp. 285-309, 2001.

[4] W. B. Liu and N. N. Yan, "A posteriori error estimates for convex boundary control problems," SIAM Journal on Numerical Analysis, vol. 39, no. 1, pp. 73-99, 2001.

[5] J. W. Zhou, Y. P. Chen, and Y. Q. Dai, "Superconvergence of triangular mixed finite elements for optimal control problems with an integral constraint," Applied Mathematics and Computation, vol. 217, no. 5, pp. 2057-2066, 2010.

[6] Y. Chen, N. Yi, and W. Liu, "A Legendre-Galerkin spectral method for optimal control problems governed by elliptic equations," SIAM Journal on Numerical Analysis, vol. 46, no. 5, pp. 2254-2275, 2008.

[7] L. Yuan and D. P. Yang, "A posteriori error estimate of optimal control problem of PDE with integral constraint for state," Journal of Computational Mathematics, vol. 27, no. 4, pp. 525542, 2009.

[8] M. Ainsworth and J. T. Oden, "A posteriori error estimation in finite element analysis," Computer Methods in Applied Mechanics and Engineering, vol. 142, no. 1-2, pp. 1-88, 1997.

[9] S. C. Brenner and L. R. Scott, The Mathematical Theory of Finite Element Methods, vol. 15, Springer, New York, NY, USA, 1994.

[10] B. Q. Guo, "Recent progress in a-posteriori error estimation for the p-version of finite element method," in Recent Advances in Adaptive, Z.-C. Shi, Z. Chen, T. Tang, and D. Yu, Eds., vol. 383 of Comtemporary Mathematics, pp. 47-61, American Mathematical Society, Providence, RI, USA, 2005.

[11] J. W. Zhou and D. P. Yang, "An improved a posteriori error estimate for the Galerkin spectral method in one dimension," Computers \& Mathematics with Applications, vol. 61, no. 2, pp. 334-340, 2011.

[12] J. W. Zhou and D. P. Yang, "Spectral mixed Galerkin method for state constrained optimal control problem governed by the first bi-harmonic equation," International Journal of Computer Mathematics, vol. 88, no. 14, pp. 2988-3011, 2011.

[13] R. A. Adams, Sobolev Spaces, Academic Press, New York, NY, USA, 1978.

[14] J. Shen, "Efficient spectral-Galerkin method. I. Direct solvers of second- and fourth-order equations using Legendre polynomials," SIAM Journal on Scientific Computing, vol. 15, no. 6, pp. 1489-1505, 1994.

[15] L. C. Evans, Partial Differential Equations, vol. 19 of Graduate Studies in Mathematics, American Mathematical Society, Providence, RI, USA, 1998. 


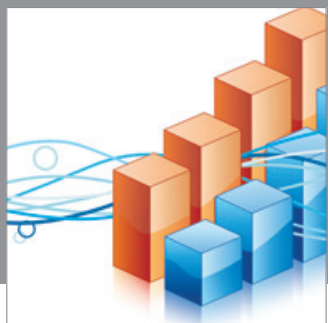

Advances in

Operations Research

mansans

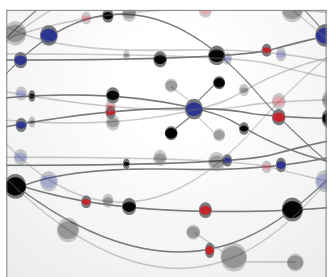

The Scientific World Journal
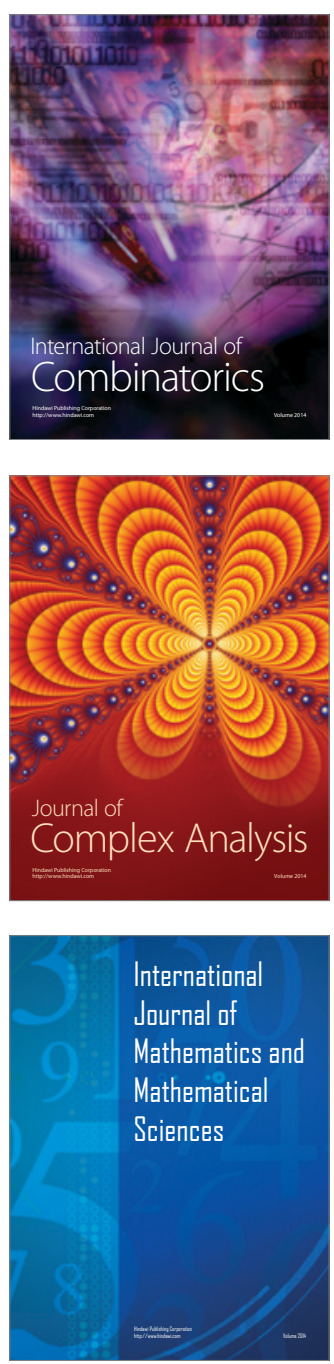
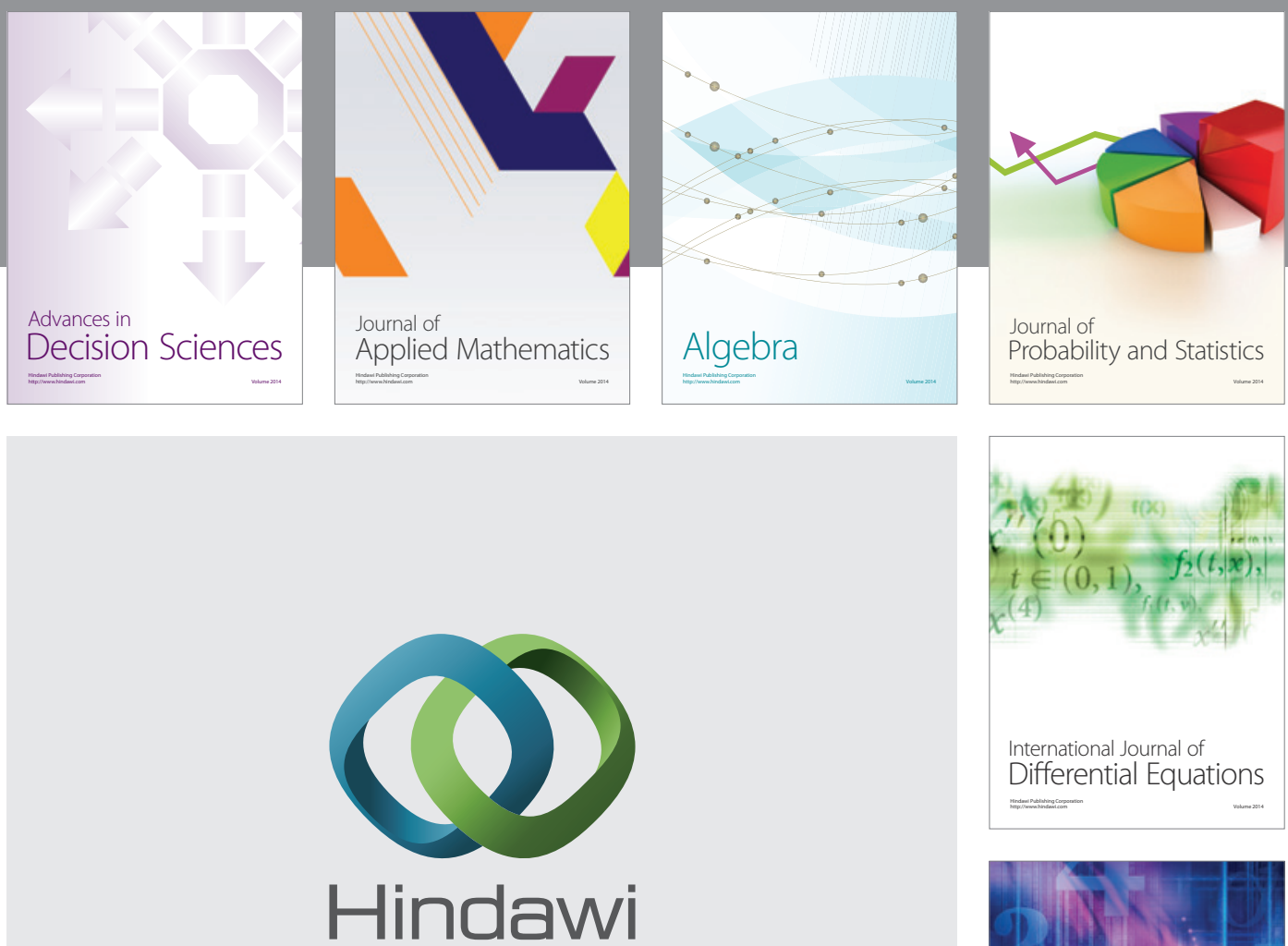

Submit your manuscripts at http://www.hindawi.com
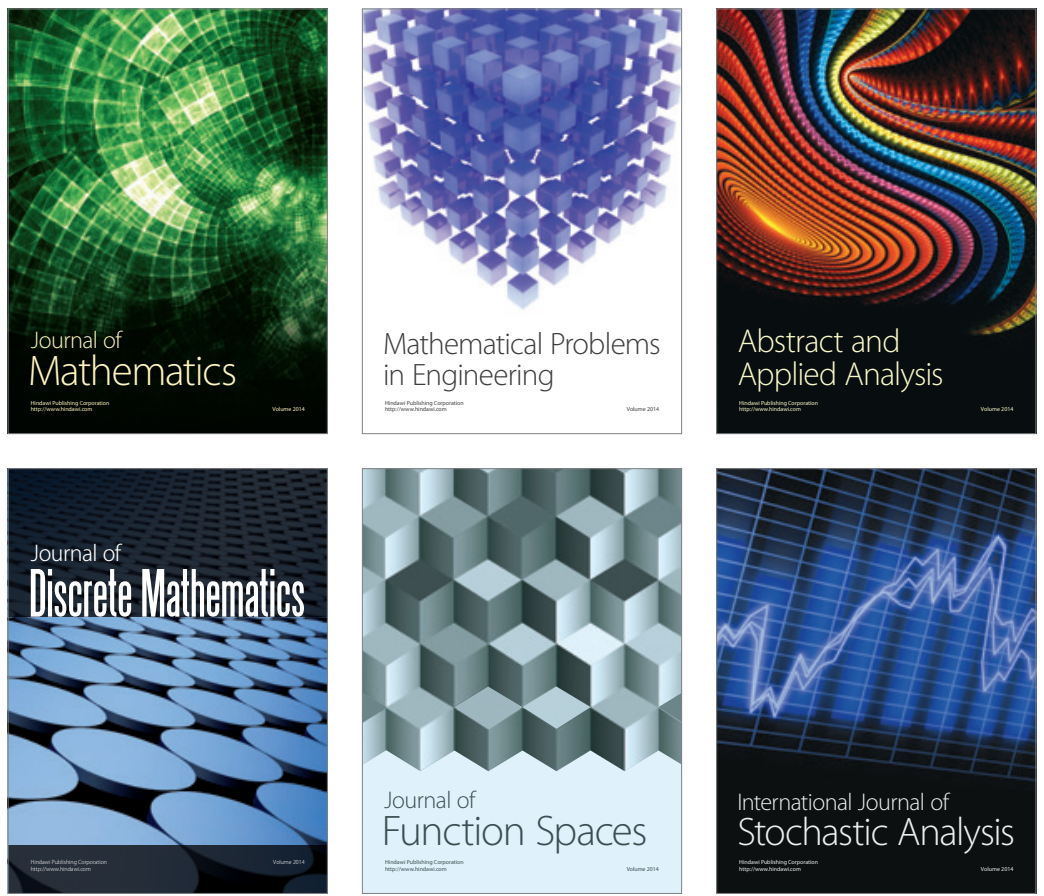

Journal of

Function Spaces

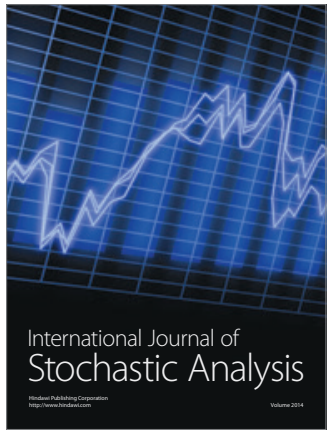

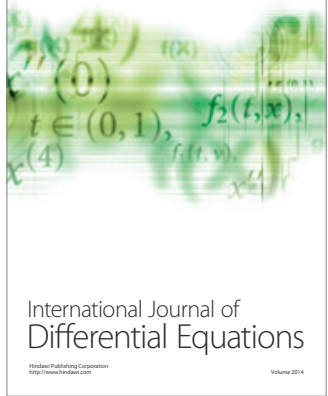
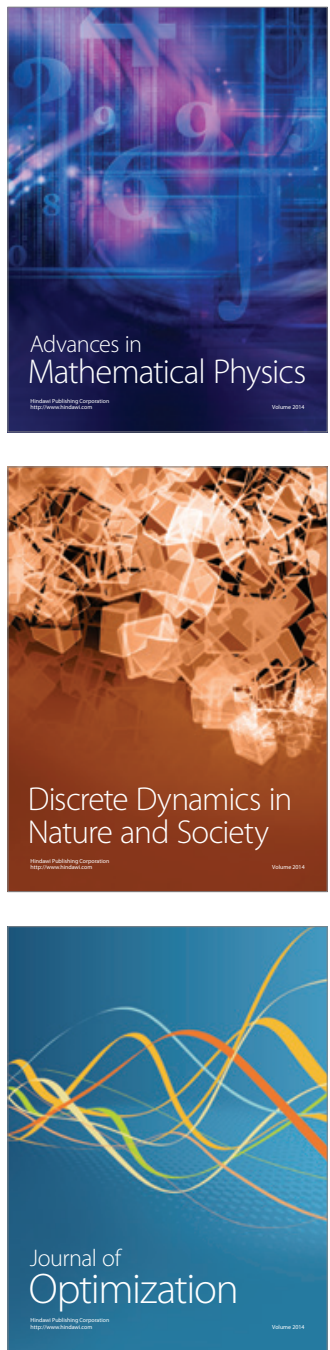